Cascading Effects Between Critical Infrastructures

\title{
Strategy of Transforming a Territory/Municipality into a Supraresilient Smart Region/City
}

\author{
Sviatoslav Timashev ${ }^{1,2,3}$ \\ ${ }^{1}$ Science \&Engng Center "Reliability and Safety of Large Systems," Ural Branch RAS, \\ Yekaterinburg, 620049 Russia \\ ${ }^{2}$ Ural Federal University, Yekaterinburg, 620014, Russia \\ ${ }^{3}$ Old Dominion University, Norfolk, VA USA
}

doi: https://doi.org/10.21467/abstracts.93.60

\begin{abstract}
ABSTRA CT
The paper describes a novel approach, based on the concept of resilience of socio-technological interdependent infrastructures comprised of (1) interdependent physical systems, (2) social infrastructure composed of employees that run the infrastructures, and the population that uses the outputs (products and services) of these systems and lives surrounded by them. This concept is applied to transforming existing territories/ municipalities, designing new cities and developing new regions into supraresilient smart regions/cities of the 21-st century based on mobility, digitization, artificial intelligence (robotization), and information technology (Io'T). The socio-technological infrastructure system of systems resilience is the ability of a complex infrastructure system to continue performing its functions in partially damaged and / or without supplies conditions, while its human infrastructure (employees and population) are experiencing loss of life, limb, health and/orpsychological stress.

Supraresilience is the hidden intrinsic ability of the human component of the socio-technological infrastructure system to react to different applied stressors in a way that exceeds its original resilience abilities (whatever they were-physical, cognitive or social). Regional supraresilience is the ability of the regional critical infrastructures to withstand, adapt, absorb, quickly recover from stresses and shocks such as natural, technological or man-made disasters, violence, conflicts, and at the same time to improve/ increase their initial vitality parameters. By facilitating supraresilience of a society, it is possible to create a smart region / city capable of sustainable development, and to achieve long-term strategic development goals of any region, including the development of human capital, improving the quality of life and competitiveness of the economy through systematic introduction of computerized services and innovative solutions. This circumstance is the absolute and the only basis for creating a supraresilient and safe smart territory. Resiliencewise acceptable risks, implemented for managing technological, industrial and environmental processes of a smart region, are fully compatible with the black swan type accidents and disasters.

For a holistic solution of the above problem an innovative comprehensive cloud IT tool that serves as a SaaS (Software as a Service) is being developed and proposed, namely, the RASOSR (resilience and safety of sustainable smart regions) software package combined with a client-tailored front-end set of sensors that provide the needed data from infrastructure components. The initial data derived from the sensors distributed throughout the regional infrastructure is analyzed following six functional and technological areas of digitalization: (1) lifestyle, (2) people, (3) the economy, (4) mobility, (5) management, and (6) environment.

For the safe implementation of the above services, RASOSR is using the Emercoin version of adapted block chain technology, during diagnostic verification (sensor data, control commands, events), monitoring and maintenance of all types of infrastructures, reliability of records, time chains of sensor and instrument readings with full trust for network participants; confirmation of damage estimates from incidents, accidents

(C) 2020 Copyright held by the author(s). Published by AIJR Publisher in "Abstracts of The Second Eurasian RISK-2020 Conference and Symposium" April 12- 19, 2020, Tbilisi, Georgia. Jointly organized by AMIR Technical Services LLC, Georgian Technical University, Institute of Geography (Kazakhstan) and Russian Institute of Petroleum Geology and Geophysics.

AijR DOI: $10.21467 /$ abstracts. 93
\end{abstract}


The Second Eurasian RISK-2020 Conference and Symposium

and disasters; record of transactions in IoT, micropayments; optimization of regional governance, cyber security; integration with RASOSR and physical subsystem of regional resilience; writing (no more than 20 $\mathrm{KB}$ /reading data in any format from any host; data exchange with block chain via http, via standardized API, locally or via remote server; allocation of geographical areas and user groups.

RASOSR provides a practical solution to the problem of actively managing regional economy by ensuring the continuity of business flow of digital services and infrastructures, as it allows to: optimize regional infrastructures short and long term safety investments according to the macroeconomic criteria of the life quality index (LQI) and willingness to pay (WTP); provide operational support to decision makers at the region/city level using the territorial average life expectancy $(A L E)$ and the regional LQI criteria; identify areas of vulnerability in a region/city; assess the depth (number of services/infrastructures involved in an accident) of potential cascading accidents; develop practical measures to prevent and mitigate the consequences of accidents and disasters; adapt regional residents to the ever-accelerating changes in digital technologies of the 21 st century and quickly stop / minimize social stressors; build a digital model of the region physical and social (supra)resilience.

Using RASOSR implies creating a live laboratory (LL) composed of: (1) The strategic Regional council of stakeholders (including the regional/municipal resilience office (RRO) with its Chief Resilient OfficerCRO); (2) Board of directors of all major / critical services and businesses in the region; (3) Implementation group of the strategic council roadmap;(4) Active supraresilient residents of the region. The LL allows establishing an empirical link between the tangible and the intangible: between the quality of regional systems operation and the well-being of region citizens. In the RRO situation room a group of regional resilience specialists provides comprehensive support for strategic and tactical decisions developed at all levels of regional government. It includes: simulating scenarios generated by the stakeholder council; creating digital (quasi) twins of the territory, municipality, village, system; ensuring continuity of business flows by managing regional resilience according to the described above RASOSR methodology; implementing breakthrough technology of regional risk management (governance) according to LQI, supraresilience, and territorial entropy. The developed methodology directly relates to MAICS-technology and infranetics [1] and links results of the theory of structures, digital fracture mechanics, risk analysis, optimization theory, demography and macroeconomics, cognitive and social sciences, as well as sciences that determine regional specifics (type of industry, biology, agriculture, tourism). Potential users of the RASOSR are local authorities, managers and emergency planners in the risk zone, providers of regional services. consultants / private companies, researchers. The RASOSR benefits: optimizes operating expenses; predicts the possibility and improves management of the elimination and mitigation of cascading accidents; ranks investments and improves integrated infrastructure management from the standpoint of supraresilience; visualizes cross-functional regional services; establishes a link between GIS and integrated information that flows within regional decision-making and policy-making processes; protects against potential climate, natural or technical impacts; improves cooperation between regional service operators; eliminates infrastructure interdependency violations; identifies areas that need increasing supraresilience (suggestions on the place of sensors installation, optimization of the investments according to the LQI criteria). The RASOSR methodology (its city-oriented version, under the brand name HAZUR) was implemented in Barcelona (Spain), Bristol (UK), Lisboa (Portugal), et al. Currently it is offered to Yekaterinburg (Russia), Almaty (Kazakhstan), Issyk-Kul region of the Kyrgyz Republic [1].

\section{Reference}

[1] S.A. Timashev. The strategy of transforming the Issyk-Kul oblast of the Kyrghyz Republic into a supraresilient smart region. Ural Branch Russian ac. of sciences, Preprint, 66p. Yekaterinburg, 2019. 